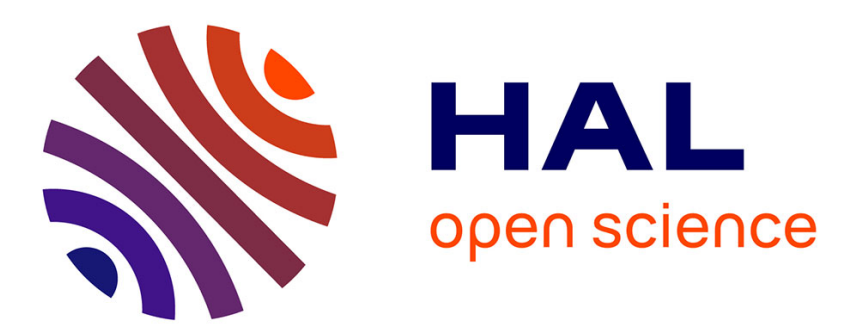

\title{
Multi-winner scoring election methods: Condorcet consistency and paradoxes
}

\author{
Mostapha Diss, Ahmed Doghmi
}

\section{To cite this version:}

Mostapha Diss, Ahmed Doghmi. Multi-winner scoring election methods: Condorcet consistency and paradoxes . 2016. halshs-01285526

\section{HAL Id: halshs-01285526 \\ https://shs.hal.science/halshs-01285526}

Preprint submitted on 9 Mar 2016

HAL is a multi-disciplinary open access archive for the deposit and dissemination of scientific research documents, whether they are published or not. The documents may come from teaching and research institutions in France or abroad, or from public or private research centers.
L'archive ouverte pluridisciplinaire HAL, est destinée au dépôt et à la diffusion de documents scientifiques de niveau recherche, publiés ou non, émanant des établissements d'enseignement et de recherche français ou étrangers, des laboratoires publics ou privés. 


\title{
GATE

UMR 5824

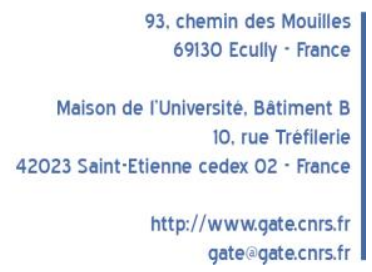

WP 1613 - March 2016

\section{Multi-winner scoring election methods: Condorcet consistency and paradoxes}

\author{
Mostapha Diss, Ahmed Doghmi
}

\begin{abstract}
:
The goal of this paper is to propose a comparison of four multi-winner voting rules, $k$-Plurality, $k$-Negative Plurality, $k$-Borda, and Bloc, which can be considered as generalisations of well-known single-winner scoring rules. The first comparison is based on the Condorcet committee efficiency which is defined as the conditional probability that a given voting rule picks out the Condorcet committee, given that such a committee exists. The second comparison is based on the likelihood of two paradoxes of committee elections: The Prior Successor Paradox and the Leaving Member Paradox which occur when a member of an elected committee leaves. In doing so, using the well-known Impartial Anonymous Culture condition, we extend the results of Kamwa and Merlin (2015) in two directions. First, our paper is concerned with the probability of the paradoxes no matter the ranking of the leaving candidate. Second, we do not only focus on the occurrence of these paradoxes when one wishes to select a committee of size $k=2$ out of $m=4$ candidates but we consider more values of $k$ and $m$.
\end{abstract}

\section{Keywords:}

Multi-winner voting rules, committee, Condorcet committee efficiency, paradoxes, probability.

JEL codes:

D71, D72

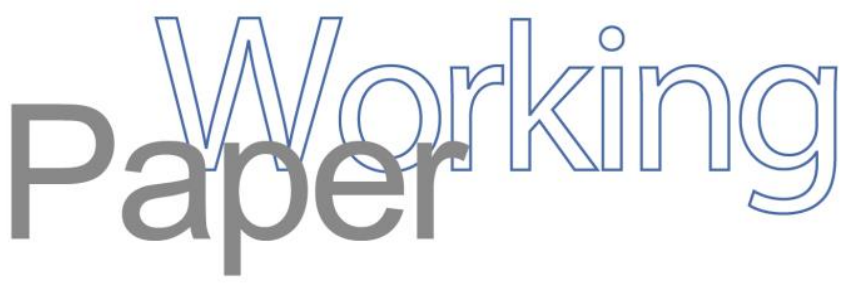




\title{
Multi-winner scoring election methods: Condorcet consistency and paradoxes
}

\author{
Mostapha Diss* $\quad$ Ahmed Doghmi ${ }^{\dagger}$
}

This version: March 8, 2016

\begin{abstract}
The goal of this paper is to propose a comparison of four multi-winner voting rules, $k$-Plurality, $k$-Negative Plurality, $k$-Borda, and Bloc, which can be considered as generalisations of well-known single-winner scoring rules. The first comparison is based on the Condorcet committee efficiency which is defined as the conditional probability that a given voting rule picks out the Condorcet committee, given that such a committee exists. The second comparison is based on the likelihood of two paradoxes of committee elections: The Prior Successor Paradox and the Leaving Member Paradox which occur when a member of an elected committee leaves. In doing so, using the well-known Impartial Anonymous Culture condition, we extend the results of Kamwa and Merlin (2015) in two directions. First, our paper is concerned with the probability of the paradoxes no matter the ranking of the leaving candidate. Second, we do not only focus on the occurrence of these paradoxes when one wishes to select a committee of size $k=2$ out of $m=4$ candidates but we consider more values of $k$ and $m$.
\end{abstract}

Keywords: Multi-winner voting rules, committee, Condorcet committee efficiency, paradoxes, probability.

JEL classification: D71, D72

\section{Introduction}

Many representative bodies in reality require the selection of a specific number of candidates (committee) instead of a single member. As for single-winner elections, there are several different ways of finding the winning committee that, in some sense, best represent all the voters. Multi-winner procedures map the preferences of the voters to subsets of candidates, and effectively specify the composition of the committee. In this setting, we need first to describe how a voter can translate his/her preference into a vote. Two main approaches are considered in the literature. In the first one, the voters have preferences over committees that satisfy certain conditions as in Fishburn (1981). In the second one, the voters are assumed to possess rankings over the candidates as single members as in Gehrlein (1985b). Whatever the case, the study of the characteristics and properties of multi-winner voting methods has received increasing interest in the recent literature of Social Choice Theory.

\footnotetext{
${ }^{*}$ Corresponding author. Univ Lyon, UJM Saint-Etienne, CNRS, GATE L-SE UMR 5824, F-42023 SaintEtienne, France. Tel.: + 33 (0)4 696611 55. Email: diss@gate.cnrs.fr.

${ }^{\dagger}$ University of Rabat, Mohammadia School of Engineering, the QSM Laboratory, Avenue Ibn Sina B.P. 765 Agdal, 10100 Rabat, Morocco. Email: ahmeddoghmi@hotmail.com.
} 
Similarly to the second setting, we consider in this paper that voters have preferences over candidates. More precisely, we assume that the voters' preferences are described by linear rankings of the set of candidates, i.e. the indifferences are not allowed. The purpose of this work is to study four multi-winner voting rules which can be considered as generalisations of well-known single-winner scoring rules: $k$-Plurality, $k$-Negative Plurality, $k$-Borda, and Bloc. Here, each candidate gets some points from each voter according to his/her position in the voter's preference, and in the case where the committee must be of size $k$, the candidates with the $k$ highest aggregate scores are elected.

First, we focus on the ability of each multi-winner rule to select the Condorcet committee à la Gehrlein (Gehrlein, 1985b). This is considered as the most important extension of Condorcet principle (Condorcet, 1785) to the $k$-winner context, when it is assumed that voters provide their preferences over candidates. The Condorcet principle considers that if a candidate beats all other candidates in pairwise majority comparisons, called Condorcet winner, then this candidate should be chosen. In this sense, Gehrlein (1985b) defined a Condorcet committee as a set of $k$ candidates such that each element of this committee defeats every candidate which does not belong to it in pairwise majority comparisons, whenever such committee exists. Consequently, we use this framework for evaluating the Condorcet committee efficiency (CCE) of each voting procedure, which is defined as the conditional probability that this procedure selects the Condorcet committee, given that a Condorcet committee exists.

There is a large literature devoted exclusively to the Condorcet efficiency of single-winner ${ }^{1}$ voting methods under various assumptions. The reader is referred to Cervone et al. (2005), Diss and Gehrlein (2015), Diss et al. (2010), Fishburn (1974a,b), Fishburn and Gehrlein (1976a,b, 1977), Gehrlein (1985a, 1995, 1999), Gehrlein and Lepelley (1999, 2001, 2015), Lepelley et al. (2000b), Merrill (1984, 1985), among others. A detailed survey of early research on the Condorcet efficiency of voting rules can be found in the recent book of Gehrlein and Lepelley (2010). However, it is striking how very little attention has been paid to the CCE. In our knowledge, the only paper concerned with such a question is the one of Gehrlein (1985b). For a number of candidates $m$ in $\{3,4,5,6\}$, this paper is concerned with the probability that a Condorcet committee exists, and the CCE of various constant scoring rules using exclusively Monte-Carlo simulations in the spirit of the well-known Impartial Culture assumption (defined later). Under a constant scoring rule, each voter shall be able to vote for a given number of candidates and the $k$ elected members of the committee are the $k$ candidates receiving the most votes ${ }^{2}$. Throughout our paper, for a number of candidates $m$ in $\{3,4,5,6\}$, we extend the previous work to more multi-winner voting rules and other contexts described later. This defines the first purpose of our paper.

Second, this study also investigates a comparison of the multi-winner rules considered in this paper on the basis of the probability that some voting paradoxes will be observed in committee elections, if a member of an elected committee leaves the competition. Two paradoxes are considered: The Prior Successor Paradox (PSP) as defined in Kamwa and Merlin (2015) and the Leaving Member Paradox (LMP) which is defined in Staring (1986) and also considered in Kamwa and Merlin (2015). Similarly to our paper, Staring

\footnotetext{
${ }^{1}$ The Condorcet efficiency of a single-winner voting rule is the conditional probability that this given voting rule picks out the Condorcet winner, given that such a candidate exists.

${ }^{2}$ To be more exact, Gehrlein (1985b) provided the probability that a Condorcet committee exists for $m$ in $\{3,4,5,6,7\}$, and the CCE of various constant scoring rules for $m$ in $\{3,4,5,6\}$.
} 
(1986) considered $n$ voters and $m$ candidates for which the election procedure selects a $k$ committee members with the $k$ greatest scores. If an elected member of a committee leaves the competition for some reason, the LMP occurs if, by holding a new election over the $m-1$ remaining candidates, the new committee and the original one are disjoint. In other words, committee members under the new election scenario are chosen, while no candidate was considered from the committee of the original election over $m$ candidates. Kamwa and Merlin (2015) defined the PSP which is less severe than the LMP. Since the elected committee members are those with the $k$ greatest scores, the authors defined the Prior Successor as the candidate with the $k+1$ th best score. Following this definition, the PSP occurs if after a member of the elected committee leaves, a new election results in a committee containing all the $k-1$ members of the original committee without the prior successor.

In spite of the importance of committee selection, there have been few studies of the occurrence of these two paradoxes under the voting schemes involved. Exceptions are Kamwa and Merlin (2015), and Staring (1986). By only examples, Staring (1986) showed that committee elections can lead to disturbingly paradoxical events similar to the LMP 3 . In particular, Staring (1986) considered that the election procedure asks each voter to vote for their $k$ most preferred candidates. Kamwa and Merlin (2015) examined the probability of the PSP and the LMP with the same four multi-winner voting rules that we consider in this paper under the IC condition when the election is concerned with a committee of $k=2$ out of $m=4$ candidates. Using the IAC condition (defined later), we enrich the results of Kamwa and Merlin (2015) in many directions. In contrast with Kamwa and Merlin (2015), our paper is concerned with the probability of the two paradoxes no matter the ranking of the leaving candidate which seems to be the natural way of considering this question. Moreover, we consider more possibilities for $k$ and $m$. This summarizes the second purpose of our study.

The rest of this paper is structured as follows. Section 2 presents the basic terminology including individual preferences and multi-winner voting rules, Section 3 states and provides the CCE of each multi-winner voting rule, Section 4 discusses the probability of the PSP and the LMP for each voting rule, and Section 5 makes some comments and conclusions.

\section{Preliminary definitions}

Before describing the voting procedures under investigation, an outline of the context, notations and assumptions is in order. Throughout the paper, we will consider the

\footnotetext{
${ }^{3}$ In addition to the LMP, the other paradox described by Staring (1986) in his original paper is the Increasing Committee Size Paradox (ICSP) which occurs if, by increasing the target size $k$, we can find that the candidates that are elected in the committees change for each $k$. Under some conditions, both committees may be totally disjoint. Notice that, for the constant scoring rule as voters cast votes for their $k$ most preferred candidates, Kamwa (2013) provided the probability of the ICSP when the size of a committee increases from 1 to 2 in threecandidate elections under the IAC condition as a function of the number of voters. Mitchell and Trumbull (1992) considered slightly more general voting methods: To select the $k$ members of the committee, each voter cast votes for their $k^{*}$ most preferred candidates. Two particular paradoxes are then considered using Monte-Carlo simulations under different assumptions, including the IC hypothesis. The first paradox is a version of the ICSP in which a candidate is elected under a vote for $k^{*}$ candidates procedure, and then that candidate is not elected for larger values of $k^{*}$. The second paradox considered in Mitchell and Trumbull (1992) occurs when the Condorcet winner is not selected among the $k$ members of the committee.
} 
framework of elections which can be defined by a pair $(X, V)$, where $X=\left\{x_{1}, \ldots, x_{m}\right\}$ is a set of candidates (or alternatives) and $V=\{1, \ldots, n\}$ is a set of voters. A linear order on $X$ is a transitive, antisymmetric, and total relation on $X$. Each voter is assumed to have a linear order on the set of candidates from the most desirable candidate to the least desirable one. In addition, each voter is assumed to act according to his/her true preferences. In this context, a profile is a sequence of $n$ total orders over $m$ candidates and a voting situation is defined by the vector $\tilde{n}=\left(n_{1}, n_{2}, \ldots, n_{m !}\right)$, where in parenthesis, we refer the number of voters endowed with each of the $m$ ! orders such that $\sum_{i=1}^{m !} n_{i}=n$. For instance, in the case of three candidates in $X=\left\{x_{1}, x_{2}, x_{3}\right\}$, there exist six possible linear orders: $x_{1} x_{2} x_{3}\left(n_{1}\right), x_{1} x_{3} x_{2}\left(n_{2}\right), x_{2} x_{1} x_{3}\left(n_{3}\right), x_{2} x_{3} x_{1}\left(n_{4}\right), x_{3} x_{1} x_{2}\left(n_{5}\right)$, and $x_{3} x_{2} x_{1}\left(n_{6}\right)$. The notation $x_{1} x_{2} x_{3}$ means that voters have candidate $x_{1}$ as most preferred, candidate $x_{3}$ as least preferred, and candidate $x_{2}$ as middle-ranked and we give in parenthesis the number $n_{1}$ of voters endowed with this order. In this setting, a voting situation is a vector $\tilde{n}=\left(n_{1}, \ldots, n_{6}\right)$ such that $\sum_{i=1}^{6} n_{i}=n$.

Given two candidates $x_{1}$ and $x_{2}$, we denote $x_{1} \mathbf{M} x_{2}$ to say that candidate $x_{1}$ beats candidate $x_{2}$ in a pairwise election which occurs when a strict majority of voters prefer $x_{1}$ to $x_{2}$. A candidate is said to be a Condorcet winner if he/she wins in all of his/her pairwise elections. For instance, using our notations above for the case of three candidates, $x_{1}$ is a Condorcet winner if $x_{1} \mathbf{M} x_{2}$ and $x_{1} \mathbf{M} x_{3}$. This is equivalent to $n_{1}+n_{2}+n_{5}>n_{3}+n_{4}+n_{6}$ and $n_{1}+n_{2}+n_{3}>n_{4}+n_{5}+n_{6}$, respectively. Clearly, each election has at most one Condorcet winner, but many elections have no Condorcet winners.

In our framework, we consider settings where the voters' goal is to elect a committee of size $k$ and we denote by $\mathcal{C}$ the set of all subsets of $X$. We can now formally define the notion of Condorcet committee à la Gehrlein ${ }^{4}$ (Gehrlein, 1985b).

Definition 1 (Condorcet committee à la Gehrlein)

$A$ set $C \subset \mathcal{C}$ is a Condorcet committee if $x_{i} \boldsymbol{M} x_{j}$ for every alternative $x_{i} \in C$ and every alternative $x_{j} \in X \backslash C$.

A multi-winner voting rule maps the preferences of all voters $V=\{1, \ldots, n\}$ to a subset $C \subset \mathcal{C}$, which consequently specifies the composition of the committee. We examine four multi-winner voting rules. The reason of considering these multi-winner voting rules is due to the fact that they are based on the general scoring protocol. A scoring rule can be expressed by a sequence of real numbers $s=\left(s_{1}, s_{2}, \ldots, s_{m}\right)$ such that $s_{1} \geq s_{2} \geq \cdots \geq s_{m}$ and $s_{1}>s_{m}$. Each of the $n$ voters ranks the candidates assigning $s_{1}$ points to the one ranked first, $s_{2}$ points to the one ranked second, and so on until $s_{m}$, the number of points assigned to the candidate ranked last. Predictably, the candidate that accumulated the most points, summed over all voters, wins the election. Since we consider settings where the voters' goal is to elect a committee of size $k$, it is natural to select the $k$ candidates with the highest scores. Given these definitions, we are ready to describe the multi-winner voting rules that we focus on in our paper.

k-Plurality: under $k$-Plurality ${ }^{5}$, we select the $k$ candidates with the highest Plurality scores such that each voter has one vote which he/she can cast for any one of the $m$ candidates, i.e. $s=(1,0, \ldots, 0)$.

\footnotetext{
${ }^{4}$ Also called weak Condorcet committee (Kaymak and Sanver, 2003) and Condorcet $k$-Committee or Condorcet $k$-Set (Pérez et al., 2012).

${ }^{5}$ Also called Single Nontransferable Vote (SNTV).
} 
k-Borda: under $k$-Borda, we select the candidates with the $k$ highest Borda scores such that each first-place vote is worth $m-1$ points, each second-place vote is worth $m-2$ points, and so on until 0 point to each last-place vote, i.e. $s=(m-1, m-2, \ldots, 1,0)$.

k-Negative Plurality: under $k$-Negative Plurality, we return the candidates with the $k$ highest Negative Plurality (also called Anti-Plurality or Veto) scores such that each voter assigns one point for any one of the $m-1$ best ranked candidates, i.e. $s=$ $(1,1, \ldots, 1,0)$.

Bloc: under $\mathrm{Bloc}^{6}$, we choose the candidates with the $k$ highest $k$-approval scores in the winning committee. $k$-approval rule is a variation of the well-known Approval Voting rule $^{7}$ obtained by fixing the number $k$ of candidates that has to be approved of by each voter. In other terms, the score of a candidate $x_{i}$ is the number of voters that rank $x_{i}$ among the top $k$ positions in a profile of strict preferences, i.e. $s=(\underbrace{1, \ldots, 1}_{k}, 0, \ldots, 0)^{8}$.

Given two candidates $x_{1}$ and $x_{2}$, we denote $x_{1} \mathbf{S} x_{2}$ to say that candidate $x_{1}$ beats candidate $x_{2}$ when the multi-winner scoring rule $\mathbf{S}$ is used. For instance, using our notations above for the case of three candidates and considering $\mathbf{S}=k$-Plurality, the event $x_{1} \mathbf{S} x_{2}$ occurs when the $k$-Plurality score of $x_{1}$ is greater than the one of $x_{2}$, which is equivalent to $n_{1}+n_{2}>n_{3}+n_{4}$.

Debord (1993) extended the Young (1975)'s characterization of Borda to the case of $k$-Borda and introduced new axioms for multi-winner rules. Elkind et al. (2015) reviewed some multi-winner voting rules and proposed a set of natural properties against which these voting rules can be judged. Among these rules, Elkind et al. (2015) are concerned with $k$-Plurality, $k$-Borda, and Bloc. Felsenthal and Maoz (1992) took into account some normative properties of four $k$-winner electoral procedures based on scoring protocols, such as $k$-Plurality rule and $k$-Borda rule.

To find our probabilities, we need to assume a probability distribution that underlies our calculations. The most common assumption is that preferences follow a uniform distribution. In this paper, the probabilities that we investigate are driven by the wellknown Impartial Anonymous Culture (IAC) condition (Gehrlein and Fishburn, 1976) which assumes that all voting situations $\tilde{n}=\left(n_{1}, n_{2}, \ldots, n_{m !}\right)$ for a specified number of voters $n$ are equally likely to be observed. This assumption is one of the most used probabilistic models in the literature. The reader is referred to Gehrlein and Lepelley (2012) for a detailed discussion of this assumption and some others such that the Impartial Culture (IC) condition $^{9}$. Gehrlein and Lepelley (2012) clarified the value of the research that is based on these simple assumptions. More precisely, the authors claimed that these conditions

\footnotetext{
${ }^{6}$ Also called Limited Voting (Kamwa and Merlin, 2015) or Constant Scoring Rule (Gehrlein, 1985b).

${ }^{7}$ Approval voting $(\mathrm{AV})$ is a voting procedure in which voters may vote for as many candidates as they wish. It asks each voter to distinguish the candidates she approves of from the ones she considers as unacceptable. The alternative with the highest degree of approbation is then selected. The proponents of this voting procedure (e.g. Brams (1980), Brams and Fishburn (1978, 2005), and Fishburn and Brams (1981)) discussed several advantages that it has over other electoral systems.

${ }^{8} \mathrm{Bloc}$ is equivalent to $k$-Plurality and $k$-Negative Plurality when $k=1$ and $k=m-1$, respectively.

${ }^{9} \mathrm{IC}$ is a second well-known model which considers the set of all preference profiles as a sample space where a voter preference profile identifies the specific preference ranking that each voter has on the candidates. In other terms, when strict preferences over the set of $m$ candidates are assumed, the IC assumption (Guilbaud, 1952 ) assumes that each voter is equally likely to pick any of the $m$ ! preferences. Notice that individual voter's preferences are not anonymous under IC condition while they are under IAC assumption.
} 
add very significant value to research on the probability that various paradoxical election outcomes might be observed. For a comprehensive survey of various probability models used in Social Choice Theory, the reader is referred to Berg and Lepelley (1994), or the recent book of Gehrlein and Lepelley (2010).

\section{The Condorcet committee efficiency}

We are now interested in the following question: Which rule has the greatest CCE? As noted in the Introduction, this question has attracted very few consideration in the literature. To the best of our knowledge, Gehrlein (1985b) is the only author who explored such framework that we extend in three main directions. First, we are concerned with more multi-winner voting rules. Second, we can provide exact results of both, the probability that a Condorcet committee exists, and the CCE when the number of candidates $m$ equal to 3 and 4 . For $m$ equal to 5 and 6 , one problem is that the number of constraints can be prohibitively large, so that in this case we will instead run Monte-Carlo simulations ${ }^{10}$. Third, we consider the IAC condition to describe the likelihood of the possible individual orderings.

It is clear that, in order to provide the CCE of each voting rule, we first need to determine the probability that a Condorcet committee (à la Gehrlein) exists.

\subsection{The probability that a Condorcet committee exists}

We begin this section by providing a general insight into the technique used in this paper in order to calculate each limiting probability under IAC condition as $n \rightarrow \infty$ for the cases of $m=3$ and $m=4$. In this framework, we will see that this technique is simply reduced to computation of volumes of convex polytopes. For this, we give a simple example with $m=3$ and $k=2$. The detail of the other calculations are available upon simple request from the authors. We begin by re-adapting our definition of a voting situation to the limiting case as $n \rightarrow \infty$. Let $p_{i}=\frac{n_{i}}{n} \geq 0$ denote the proportion of the $n$ voters with the associated $i$ th linear ordering for $1 \leq i \leq 6$ in a given election with a large electorates. In other words, there exist six possible linear orders: $x_{1} x_{2} x_{3}\left(p_{1}\right), x_{1} x_{3} x_{2}\left(p_{2}\right), x_{2} x_{1} x_{3}\left(p_{3}\right), x_{2} x_{3} x_{1}\left(p_{4}\right), x_{3} x_{1} x_{2}\left(p_{5}\right)$, and $x_{3} x_{2} x_{1}\left(p_{6}\right)$. Any voting situation is completely specified by a six-dimensional vector $\tilde{p}=\left(p_{1}, \ldots, p_{6}\right)$ such that $\sum_{i=1}^{6} p_{i}=1$. Similarly, the IAC condition, henceforth referred to as $\mathrm{IAC}_{\infty}$, is equivalent to assuming that all possible $\tilde{p}$ are equally likely to be observed. It is clear that all the possible voting situations $\tilde{p}$ are included in the convex polytope defined by the following six vertices:

$$
\begin{array}{llrl}
v_{1}^{1}=[1,0,0,0,0,0] & v_{2}^{1}=[0,1,0,0,0,0] & v_{3}^{1}=[0,0,1,0,0,0] \\
v_{4}^{1}=[0,0,0,1,0,0] & v_{5}^{1}=[0,0,0,0,1,0] & v_{6}^{1}=[0,0,0,0,0,1]
\end{array}
$$

This region corresponds to a five-dimensional simplex $\Delta^{5}$. The first objective is to find the volume of this region. Several methods or algorithms can be found for volume computations of polytopes. The reader is referred to Bueler et al. (2000), Cervone et al. (2005), Cohen and Hickey (1979), Lawrence (1991), or Lepelley et al. (2008). Details of the procedure used for our calculations are not included in the current paper, and we only present an outline of

\footnotetext{
${ }^{10}$ We can also generate simulations with more than six candidates. However, this option is ignored since these simulations can not affect the conclusions of our paper.
} 
how the results are obtained ${ }^{11}$. Using the same general procedure that was implemented in Cervone et al. (2005), we can obtain the volume of this space which is also provided in Diss and Gehrlein (2012, 2015):

$$
\operatorname{Volume}(I A C, m=3)=\frac{\sqrt{6}}{120} .
$$

Notice that for $m=4$, any voting situation $\tilde{n}=\left(n_{1}, \ldots, n_{24}\right)$ will be rewritten as $\tilde{p}=$ $\left(p_{1}, \ldots, p_{24}\right)$ such that $\sum_{i=1}^{24} p_{i}=1$. The set of all voting situations $\tilde{p}$ is then 23 -dimensional. For this region, we obtain the following volume:

$$
\operatorname{Volume}(I A C, m=4)=\frac{\sqrt{6}}{12926008369442488320000} .
$$

According to Definition 1 , a set $C \subset \mathcal{C}$ is a Condorcet committee $\grave{a}$ la Gehrlein if $x_{i} \mathbf{M} x_{j}$ for every alternative $x_{i}$ in $C$ and every alternative $x_{j}$ in $X \backslash C$. With $m=3$ and $k=2$, suppose without loss of generality that the committee is $C=\left\{x_{1}, x_{2}\right\}$. Then, $C$ is a Condorcet committee if both $x_{1}$ and $x_{2}$ defeat $x_{3}$ head-to-head. In other terms, we need the following conditions: $p_{1}+p_{2}+p_{3}>p_{4}+p_{5}+p_{6}\left(x_{1} \mathbf{M} x_{3}\right)$ and $p_{1}+p_{3}+p_{4}>p_{2}+p_{5}+p_{6}\left(x_{2} \mathbf{M} x_{3}\right)$. Thus, our objective is to obtain the polytope included in $\Delta^{5}$ for which the two inequalities are fulfilled. The procedure of obtaining the required polytope is recursive. More precisely, since each polytope is defined by its vertices, on each step the polytope obtained on the previous step is cut with the next hyperplane and its vertices are determined. Let $H_{1}$ denote the hyperplane defined from the first inequality above such that:

$$
H_{1}: p_{1}+p_{2}+p_{3}-p_{4}-p_{5}-p_{6}=0
$$

Thus, when the first inequality is taken into consideration, the original five-dimensional simplex $\Delta^{5}$ given in (1) is cut by $H_{1}$ into two convex polytopes, the one for which $x_{1} \mathbf{M} x_{3}$ $\left(p_{1}+p_{2}+p_{3}-p_{4}-p_{5}-p_{6}>0\right.$ and the other for which $x_{3} \mathbf{M} x_{1}\left(p_{1}+p_{2}+p_{3}-p_{4}-p_{5}-p_{6}<0\right)$. This second polytope is discarded along with all vertices that are included in it. After this first slice, the vertices of the new polytope include all vertices of $\Delta^{5}$ that are above or on the hyperplane $H_{1}$ plus the new vertices that appear when an edge of $\Delta^{5}$ (formed by one vertex above and one below the hyperplane $H_{1}$ ) is sliced by $H_{1}$. We can determine the vertices that remain within the region and all of the new vertices that are created after this first slice. These vertices are:

$$
\begin{array}{lll}
v_{1}^{2}=[1,0,0,0,0,0] & v_{2}^{2}=[0,1,0,0,0,0] & v_{3}^{2}=[0,0,1,0,0,0] \\
v_{4}^{2}=\left[\frac{1}{2}, 0,0,0,0, \frac{1}{2}\right] & v_{5}^{2}=\left[0, \frac{1}{2}, 0,0,0, \frac{1}{2}\right] & v_{6}^{2}=\left[0,0, \frac{1}{2}, 0,0, \frac{1}{2}\right] \\
v_{7}^{2}=\left[\frac{1}{2}, 0,0, \frac{1}{2}, 0,0\right] & v_{8}^{2}=\left[0, \frac{1}{2}, 0, \frac{1}{2}, 0,0\right] & v_{9}^{2}=\left[0,0, \frac{1}{2}, \frac{1}{2}, 0,0\right] \\
v_{10}^{2}=\left[\frac{1}{2}, 0,0,0, \frac{1}{2}, 0\right] & v_{11}^{2}=\left[0, \frac{1}{2}, 0,0, \frac{1}{2}, 0\right] & v_{12}^{2}=\left[0,0, \frac{1}{2}, 0, \frac{1}{2}, 0\right]
\end{array}
$$

Thus, when the second inequality $\left(x_{2} \mathbf{M} x_{3}: p_{1}-p_{2}+p_{3}+p_{4}-p_{5}-p_{6}>0\right)$ is taken into consideration, the new polytope is similarly defined by the following vertices:

$$
\begin{array}{llr}
v_{1}^{3}=\left[0, \frac{1}{2}, 0, \frac{1}{2}, 0,0\right] & v_{2}^{3}=\left[\frac{1}{2}, 0,0,0,0, \frac{1}{2}\right] & v_{3}^{3}=\left[0,0, \frac{1}{2}, 0,0, \frac{1}{2}\right] \\
v_{4}^{3}=\left[\frac{1}{2}, 0,0, \frac{1}{2}, 0,0\right] & v_{5}^{3}=\left[0,0, \frac{1}{2}, \frac{1}{2}, 0,0\right] & v_{6}^{3}=\left[\frac{1}{2}, \frac{1}{2}, 0,0,0,0\right] \\
v_{7}^{3}=\left[0, \frac{1}{2}, \frac{1}{2}, 0,0,0\right] & v_{8}^{3}=\left[\frac{1}{2}, 0,0,0, \frac{1}{2}, 0\right] & v_{9}^{3}=\left[0,0, \frac{1}{2}, 0, \frac{1}{2}, 0\right] \\
v_{10}^{3}=[1,0,0,0,0,0] & v_{11}^{3}=[0,0,1,0,0,0] &
\end{array}
$$

\footnotetext{
${ }^{11}$ Our volumes are found with the use of the algorithm implemented in Gawrilow and Joswig (2000).
} 
Having our final polytope, the next step is to calculate its volume. We obtain the following result:

$$
\text { Volume }_{1}=\frac{\sqrt{6}}{384} \text {. }
$$

Using the symmetry of IAC with respect to the three candidates, it then follows from (2) and (6) that the probability for which a Condorcet committee of size 2 exists in the case of 3 candidates with IAC and large electorates (denoted $P_{I A C_{\infty}}^{C C}(2,3)$ ), is given by:

$$
P_{I A C_{\infty}}^{C C}(2,3)=\frac{3 \times \text { Volume }_{1}}{\text { Volume }(I A C, m=3)}=\frac{15}{16}=0.9375 .
$$

This method is used to find all our probabilities in the case of $m=3$ and $m=4$. However, it turns impossible to obtain results in an acceptable time for $m=5$ and $m=6$. Consequently, to circumvent the impossibility of having exact results ${ }^{12}$, we perform a computer simulation to estimate these probabilities using the Monte-Carlo simulation methodology in the spirit of the IAC assumption. More exactly, we take into consideration a number of voters $n=$ 100,000 and we run 100,000 elections to guarantee our results with a very law margin of error. Computed values of $P_{I A C_{\infty}}^{C C}(k, m)$ are listed in Table 1 for the values of $m$ and $k$ considered in this paper.

Table 1: $P_{I A C_{\infty}}^{C C}(k, m)$

\begin{tabular}{l|cccc}
\hline $\begin{array}{l}m \rightarrow \\
k \downarrow\end{array}$ & 3 & 4 & 5 & 6 \\
\hline 1 & 0.9375 & 0.8384 & 0.7470 & 0.6774 \\
2 & 0.9375 & 0.7598 & 0.6120 & 0.4830 \\
3 & - & 0.8384 & 0.6020 & 0.4260 \\
4 & - & - & 0.7525 & 0.4935 \\
5 & - & - & - & 0.6828
\end{tabular}

The probability $P_{I A C_{\infty}}^{C C}(1,3)=0.9375$ is the well-known result given in Gehrlein and Fishburn (1976) that a Condorcet winner exists under IAC for $m=3$ and $n \rightarrow \infty$. The precise limiting probability $P_{I A C_{\infty}}^{C C}(1,4)=0.8384$ is similar to the one given in Gehrlein (2001). Going deeper in these probabilities, we can deduce the following facts:

- It becomes very apparent that an important result can be observed: $C C_{\infty}^{I A C}(k, m)=$ $C C_{\infty}^{I A C}(m-k, m)$. This general result is also given in Gehrlein (1985b) under IC and remains true in our context of IAC. Notice that this result has a specific interpretation when $k=1$ which is well-known from the literature: The probability that a Condorcet winner exists is identical to the probability that a Condorcet loser exists ${ }^{13}$.

- As well as $k$ increases, the probability for which a Condorcet committee exists first decreases and then increases.

- The results in Table 1 contain some information that allows us to check the margin of error of our simulation method for $m=5$ and $m=6$. Indeed, our results guarantee a maximum error of about 0.01 which corresponds to $C C_{\infty}^{I A C}(4,6)-C C_{\infty}^{I A C}(2,6)=$ $0.4935-0.4830=0.0105$.

\footnotetext{
${ }^{12}$ The only volume that we can provide in an acceptable time is the one related to the 119-dimensional region which defines all voting situations $\tilde{p}$ in the case of $m=5$ : Volume $(I A C, m=5)=1.965069984 \times 10^{-196}$.

${ }^{13} \mathrm{~A}$ Condorcet loser is a candidate that loses all head-to-head comparisons with other candidates.
} 


\subsection{The Condorcet committee efficiency of simple voting rules}

Recall that, we suppose in our illustrative example that $X=\left\{x_{1}, x_{2}, x_{3}\right\}$ and that the elected committee is $C=\left\{x_{1}, x_{2}\right\}$. Then, $C$ should be the winner when we consider a voting rule $\mathbf{S}$, i.e. $x_{1} \mathbf{S} x_{3}$ and $x_{2} \mathbf{S} x_{3}$. Let consider that $\mathbf{S}$ is equivalent to $k$-Plurality rule. Therefore, the inequalities $p_{1}+p_{2}>p_{5}+p_{6}\left(x_{1} \mathbf{S} x_{3}\right)$ and $p_{3}+p_{4}>p_{5}+p_{6}\left(x_{2} \mathbf{S} x_{3}\right)$ should be satisfied. Thus, when the first inequality is taken into consideration, the final polytope defined in (5) is discarded along with all vertices that are included in it. After this first slice the vertices of the new polytope are found. Thus, when the last inequality is taken into consideration, our final polytope, where the set $C=\left\{x_{1}, x_{2}\right\}$ is the Condorcet committee and also the winner of $k$-Plurality, is defined by the following vertices:

$$
\begin{array}{llll}
v_{1}^{4}=\left[0,0, \frac{1}{2}, \frac{1}{2}, 0,0\right] & v_{2}^{4}=\left[0, \frac{1}{2}, 0, \frac{1}{2}, 0,0\right] & v_{3}^{4}=[0,0,1,0,0,0] \\
v_{4}^{4}=\left[\frac{1}{2}, \frac{1}{2}, 0,0,0,0\right] & v_{5}^{4}=[1,0,0,0,0,0] & v_{6}^{4}=\left[0, \frac{1}{4}, \frac{1}{4}, \frac{1}{4}, 0, \frac{1}{4}\right] \\
v_{7}^{4}=\left[\frac{1}{6}, \frac{1}{6}, \frac{1}{6}, \frac{1}{6}, 0, \frac{1}{3}\right] & v_{8}^{4}=\left[\frac{1}{3}, 0, \frac{1}{6}, \frac{1}{6}, 0, \frac{1}{3}\right] & v_{9}^{4}=\left[\frac{1}{4}, \frac{1}{4}, 0, \frac{1}{4}, 0, \frac{1}{4}\right] \\
v_{10}^{4}=\left[\frac{1}{2}, 0,0, \frac{1}{4}, 0, \frac{1}{4}\right] & v_{11}^{4}=\left[0, \frac{1}{4}, \frac{1}{2}, 0,0, \frac{1}{4}\right] & v_{12}^{4}=\left[\frac{1}{6}, \frac{1}{6}, \frac{1}{3}, 0,0, \frac{1}{3}\right] \\
v_{13}^{4}=\left[\frac{1}{3}, 0, \frac{1}{3}, 0,0, \frac{1}{3}\right] & v_{14}^{4}=\left[\frac{1}{2}, 0,0, \frac{1}{2}, 0,0\right] & v_{15}^{4}=\left[0, \frac{1}{2}, \frac{1}{2}, 0,0,0\right] \\
v_{16}^{4}=\left[0, \frac{1}{4}, \frac{1}{4}, \frac{1}{4}, \frac{1}{4}, 0\right] & v_{17}^{4}=\left[\frac{1}{6}, \frac{1}{6}, \frac{1}{6}, \frac{1}{6}, \frac{1}{3}, 0\right] & v_{18}^{4}=\left[\frac{1}{3}, 0, \frac{1}{6}, \frac{1}{6}, \frac{1}{3}, 0\right] \\
v_{19}^{4}=\left[\frac{1}{4}, \frac{1}{4}, 0, \frac{1}{4}, \frac{1}{4}, 0\right] & v_{20}^{4}=\left[\frac{1}{2}, 0,0, \frac{1}{4}, \frac{1}{4}, 0\right] & v_{21}^{4}=\left[0, \frac{1}{4}, \frac{1}{2}, 0, \frac{1}{4}, 0\right] \\
v_{22}^{4}=\left[\frac{1}{6}, \frac{1}{6}, \frac{1}{3}, 0, \frac{1}{3}, 0\right] & v_{23}^{4}=\left[\frac{1}{3}, 0, \frac{1}{3}, 0, \frac{1}{3}, 0\right] &
\end{array}
$$

The volume associated to the polytope defined by these vertices is given by:

$$
\text { Volume }_{2}=\frac{17 \times \sqrt{6}}{10368} .
$$

We conclude that the Condorcet committee efficiency of $k$-Plurality for $k=2$ and $m=3$ under the IAC condition and large electorates (denoted $C C E_{I A C_{\infty}}^{k-P}(2,3)$ ), is given by:

$$
C C E_{I A C_{\infty}}^{k-P}(2,3)=\frac{\text { Volume }_{2}}{\text { Volume }_{1}}=0.6296 .
$$

Tables 2-5 display the values of $C C E_{I A C_{\infty}}^{k-P}(k, m), C C E_{I A C_{\infty}}^{k-N P}(k, m), C C E_{I A C_{\infty}}^{k-B}(k, m)$, and $C C E_{I A C_{\infty}}^{B}(k, m)$, the CCE of $k$-Plurality, $k$-Negative Plurality, $k$-Borda, and Bloc, respectively. Again, while the values for $m=3$ and $m=4$ are all exact probabilities, the ones for $m=5$ and $m=6$ are based on computer simulations.

Table 2: $C C E_{I A C_{\infty}}^{k-P}(k, m)$

\begin{tabular}{c|cccc}
\hline $\begin{array}{c}m \rightarrow \\
k \downarrow\end{array}$ & 3 & 4 & 5 & 6 \\
\hline 1 & 0.8815 & 0.7426 & 0.6143 & 0.5207 \\
2 & 0.6296 & 0.5427 & 0.4476 & 0.3638 \\
3 & - & 0.5516 & 0.4199 & 0.3380 \\
4 & - & - & 0.5100 & 0.3322 \\
5 & - & - & - & 0.4701
\end{tabular}

Table 3: $C C E_{I A C_{\infty}}^{k-N P}(k, m)$

\begin{tabular}{c|cccc}
\hline $\begin{array}{c}m \rightarrow \\
k \downarrow\end{array}$ & 3 & 4 & 5 & 6 \\
\hline 1 & 0.6296 & 0.5516 & 0.5104 & 0.4696 \\
2 & 0.8815 & 0.5427 & 0.4267 & 0.3529 \\
3 & - & 0.7426 & 0.4521 & 0.3211 \\
4 & - & - & 0.6140 & 0.3642 \\
5 & - & - & - & 0.5183
\end{tabular}


Table 4: $C C E_{I A C_{\infty}}^{k-B}(k, m)$

\begin{tabular}{c|cccc}
\hline $\begin{array}{c}m \rightarrow \\
k \downarrow\end{array}$ & 3 & 4 & 5 & 6 \\
\hline 1 & 0.9111 & 0.8706 & 0.8580 & 0.8457 \\
2 & 0.9111 & 0.8598 & 0.8286 & 0.8234 \\
3 & - & 0.8706 & 0.8348 & 0.8172 \\
4 & - & - & 0.8587 & 0.8372 \\
5 & - & - & - & 0.8477
\end{tabular}

Table 5: $C C E_{I A C_{\infty}}^{B}(k, m)$

\begin{tabular}{c|cccc}
\hline $\begin{array}{c}m \rightarrow \\
k \downarrow\end{array}$ & 3 & 4 & 5 & 6 \\
\hline 1 & 0.8815 & 0.7426 & 0.6143 & 0.5207 \\
2 & 0.8815 & 0.7468 & 0.6210 & 0.5386 \\
3 & - & 0.7426 & 0.6337 & 0.5715 \\
4 & - & - & 0.6140 & 0.5330 \\
5 & - & - & - & 0.5183
\end{tabular}

We can deduce the following facts from these probabilities:

- An immediate consequence of these tables is that our calculations are consistent with previous results in the literature when $k=1$. For instance, for $m$ between 3 and 8, Lepelley et al. (2000a) considered the Condorcet efficiency of Plurality, Negative Plurality and Borda for the asymptotic case using Monte-Carlo simulations. For instance, under IAC, the Condorcet efficiency estimates for Plurality are 0.8816, $0.7429,0.6139$, and 0.5198 for $m$ equal to $3,4,5$, and 6 , respectively while our results provide $0.8815,0.7426,0.6143$, and 0.5207 . Our results show a small difference in comparison with those provided in Lepelley et al. (2000a) for each voting rule. For $m=3$ and $k=1$, our results for Borda, Plurality, and Negative Plurality are consistent with previous exact results in Cervone et al. (2005).

- It is obvious from these tables that, for a given $k$, the CCE decreases as $m$ increases. Moreover, for a given $m$, we observe that the CCE of $k$-Negative Plurality, $k$-Plurality, and $k$-Borda exhibit the same behaviour, i.e. it first decreases and then increases as $k$ increases. However, this conclusion is not the same for Bloc since its CCE first decreases and then increases as $k$ increases.

- $k$-Borda performs better than the three other voting rules and $k$-Negative Plurality is the worst scenario. Moreover, for a given $m$, the CCE of $k$-Borda varies much more slowly than the three other rules and remains at a relatively high level when $k$ changes. For instance, for $m=6$, the probability under $k$-Borda varies between 0.8172 for $k=3$ and 0.8477 for $k=5$ while the corresponding one of $k$-Plurality ranges between 0.3322 for $k=4$ and 0.5207 for $k=1$.

- Some of the relationships which were developed in Gehrlein (1985b) under IC can be generalized to IAC. In particular, $C C E_{I A C_{\infty}}^{k-P}(k, m)$ is the same as $C C E_{I A C_{\infty}}^{k-N P}(m-k, m)$. Moreover, for Bloc we have $C C E_{I A C_{\infty}}^{B}(k, m)=C C E_{I A C_{\infty}}^{B}(m-k, m)$. Notice that the paper of Gehrlein (1985b) is not concerned in $k$-Borda for which we found that the CCE exhibits the same behaviour as Bloc.

\section{Two paradoxes with simple voting rules}

As already mentioned, this paper also extends the results of Kamwa and Merlin (2015) and discusses the probability of two paradoxes that manifested themselves in a particular context: The election of committees when a member of an elected committee leaves. We are interested in the following question: Which multi-winner voting rule has the smallest probability of each paradox? 


\subsection{The context}

Consider an election in which a committee $C=\left\{x_{1}, x_{2}, \ldots, x_{k}\right\}$ of size $k$ out of $m$ candidates is elected. Recall that, in our framework, the set $C$ is composed by the $k$ candidates with the $k$ greatest scores which means that $x_{i} \mathbf{S} x_{j}$ for each $x_{i} \in C$ and each element $x_{j} \notin C$. Suppose that a candidate $x_{l}$ is the $(k+1)$ th ranked candidate, called the Prior Successor, which happens if $x_{l} \mathbf{S} x_{j}$ for each $x_{j} \notin C$ with $x_{l} \neq x_{j}$. Then, without loss of generality, suppose that candidate $x_{1} \in C$ must immediately drop out of office for some reason and that there is no provision of substitutes. It seems very reasonable to simply nominate candidate $x_{l}$ to the committee in order to replace $x_{1}$ since $x_{l}$ was ranked $(k+1)$ th in total votes received by each candidate. This would result in the elected committee composed of $C \backslash x_{1}$ and $x_{l}$.

However, two other options would be possible and can lead to different committees. The first option would be to remove $x_{1}$ and then hold a new election over the remaining $m-1$ candidates. This case will be called New Ballot (NB). The second option would be to remove $x_{1}$ and then hold a new election over the remaining $m-k$ candidates not in the committee $C$. This case will be referred to as Partial Ballot (PB). By holding an election with all the $m-1$ candidates (NB) or another election over the $m-k$ candidates not in the committee $C$ (PB), the new committee can be composed of the $k-1$ members of the previous committee plus another candidate without the candidate $x_{l}$. This is the definition of the Prior Successor Paradox (PSP) as given in Kamwa and Merlin (2015).

Now, suppose again that a committee $C=\left\{x_{1}, x_{2}, \ldots, x_{k}\right\}$ of size $k$ out of $m$ candidates is elected and that a candidate $x_{1}$ leaves the office for some reason. By holding a new election, the candidates whom become the new elected committee members can be totally different in comparison with candidates in the original committee. This defines the Leaving Member Paradox (LMP) as introduced in Staring (1986). It is obvious that this paradox only occurs when the new election holds over the remaining $m-1$ candidates (NB).

Kamwa and Merlin (2015) considered the probability of these two paradoxes for the four multi-winner voting rules presented in this paper under the IC assumption as the number of voters tends to infinity. Using the IAC condition, we extend the results of Kamwa and Merlin (2015) in two main directions.

First, our paper is concerned with the probability of the two paradoxes no matter the ranking of the leaving candidate. Kamwa and Merlin (2015) started with considering the total collective rankings over all the candidates which consequently provide the $k$ committee members. Then, given a collective ranking over the $m$ candidates and after one candidate is removed from the competition ${ }^{14}$, the authors are interested in the probability of each possible collective ranking over the $m-1$ candidates. On this basis, the authors studied the probability of each paradox. More precisely, Kamwa and Merlin (2015) are concerned in the case of $m=4$ and $k=2$. For $X=\left\{x_{1}, x_{2}, x_{3}, x_{4}\right\}$, given that the collective ranking is $x_{1} x_{2} x_{3} x_{4}$ (which means that the committee members are $x_{1}$ and $x_{2}$ ), the authors provided two different probabilities for the PSP under NB.

$$
\begin{aligned}
& P_{P S P_{1}}^{N B}=P\left(x_{2} x_{4} x_{3} \mid \widehat{x}_{1} x_{2} x_{3} x_{4}\right)+P\left(x_{4} x_{2} x_{3} \mid \widehat{x}_{1} x_{2} x_{3} x_{4}\right) \\
& P_{P S P_{2}}^{N B}=P\left(x_{1} x_{4} x_{3} \mid x_{1} \widehat{x}_{2} x_{3} x_{4}\right)+P\left(x_{4} x_{1} x_{3} \mid x_{1} \widehat{x}_{2} x_{3} x_{4}\right)
\end{aligned}
$$

\footnotetext{
${ }^{14}$ In this framework, one supposes that voters keep their preferences unchanged over the rest of candidates no matter the candidate who leaves the elected committee.
} 
Under the original collective ranking $x_{1} x_{2} x_{3} x_{4}$ and NB, the first probability $P_{P S P_{1}}^{N B}$ is given when the candidate ranked first, i.e. $x_{1}$, leaves (noted $\widehat{x}_{1}$ ). The PSP will occur if the new collective ranking over the three remaining candidates is $x_{2} x_{4} x_{3}$ or $x_{4} x_{2} x_{3}$ which means that the two new elected members of the committee are $x_{2}$ and $x_{4}$. The second probability $P_{P S P_{2}}^{N B}$ is found when the candidate ranked second, i.e. $x_{2}$, leaves and the PSP will occur if the new collective ranking is $x_{1} x_{4} x_{3}$ or $x_{4} x_{1} x_{3}$ which means that the two new elected members of the committee are $x_{1}$ and $x_{4}$.

For $\mathrm{PB}$ and the same original collective ranking $x_{1} x_{2} x_{3} x_{4}$, the first probability $P_{P S P_{1}}^{P B}$ is given when $x_{1}$ leaves and the PSP will occur if the partial election between the two candidates not in the set $C$ leads to the event $x_{4} \mathbf{M} x_{3}$, which means that the two new elected members of the committee are $x_{2}$ and $x_{4}$. The second probability $P_{P S P_{2}}^{P B}$ is given when $x_{2}$ leaves and the PSP will occur if $x_{4}$ beats $x_{3}$ in pairwise comparison, i.e. the two new elected members of the committee are $x_{1}$ and $x_{4}$.

$$
\begin{aligned}
& P_{P S P_{1}}^{P B}=P\left(x_{4} \mathbf{M} x_{3} \mid \widehat{x}_{1} x_{2} x_{3} x_{4}\right) \\
& P_{P S P_{2}}^{P B}=P\left(x_{4} \mathbf{M} x_{3} \mid x_{1} \widehat{x}_{2} x_{3} x_{4}\right)
\end{aligned}
$$

Similarly, Kamwa and Merlin (2015) provided two different probabilities for the LMP. The first probability $P_{L M P_{1}}^{N B}$ is the one defined when $x_{1}$ drops and the second one $P_{L M P_{2}}^{N B}$ corresponds to the event when $x_{2}$ leaves, given that the original collective ranking is $x_{1} x_{2} x_{3} x_{4}$.

$$
\begin{aligned}
& P_{L M P_{1}}^{N B}=P\left(x_{4} x_{3} x_{2} \mid \widehat{x}_{1} x_{2} x_{3} x_{4}\right)+P\left(x_{3} x_{4} x_{2} \mid \widehat{x}_{1} x_{2} x_{3} x_{4}\right) \\
& P_{L M P_{2}}^{N B}=P\left(x_{4} x_{3} x_{1} \mid x_{1} \widehat{x}_{2} x_{3} x_{4}\right)+P\left(x_{3} x_{4} x_{1} \mid x_{1} \widehat{x}_{2} x_{3} x_{4}\right)
\end{aligned}
$$

The approach described by the authors has an important limit since they do not provide the joint probabilities of the events for each paradox ${ }^{15}$. As a consequence, the total probability of each paradox is not provided by the authors. As mentioned previously, in our paper, we develop an alternative representation since our probabilities are found no matter the ranking of the leaving candidate of the original committee. By doing so, we provide the total probability of each paradox.

The second contribution of this section is that we consider more values of $k$ and $m$ since we do not only focus on the paradoxes that can occur when one wishes to select a committee of size $k=2$ out of $m=4$ candidates. Kamwa and Merlin (2015) results established the superiority of $k$-Borda among the four studied voting rule when one wishes to minimize the probability of the two paradoxes. By estimating the likelihood of these paradoxes for more values of $m$ and $k$, the current section also wishes to examine whether the pre-eminence of $k$-Borda will be valid again. The last purpose of the current section is to capture how the probabilities change as a function of $m$ and $k$ under each voting rule. Again, while the corresponding probabilities for $m=3$ and $m=4$ are all exact, the ones for $m=5$ and $m=6$ are based on computer simulations.

\subsection{The prior successor paradox}

In order to highlight the difference between our approach and the one developed in Kamwa and Merlin (2015), suppose similarly that we want to elect a committee of 2 members out

\footnotetext{
${ }^{15}$ This is important since for each paradox the event describing the first probability and the second one are not disjoint.
} 
of 4 candidates in the set $X=\left\{x_{1}, x_{2}, x_{3}, x_{4}\right\}$. Without loss of generality, suppose that the elected committee is $C=\left\{x_{1}, x_{2}\right\}$ and that the prior successor is $x_{3}$ which happen when $x_{1} \mathbf{S} x_{3}, x_{1} \mathbf{S} x_{4}, x_{2} \mathbf{S} x_{3}, x_{2} \mathbf{S} x_{4}$, and $x_{3} \mathbf{S} x_{4}$ under a given voting rule $\mathbf{S}$. As a consequence, in contrast with Kamwa and Merlin (2015), we are not interested in the ranking between $x_{1}$ and $x_{2}{ }^{16}$. To summarize, the event for which the elected committee is $C=\left\{x_{1}, x_{2}\right\}$ with $x_{3}$ as a prior successor requires five inequalities to be satisfied. Using the definition of a voting situation $\tilde{p}=\left(p_{1}, \ldots, p_{24}\right)$ such that $\sum_{i=1}^{24} p_{i}=1$, our first objective is to define the volume of this space, for which we use the same general procedure of Section 3. Without loss of generality, suppose that $x_{1}$ leaves the competition and that voters keep their preferences unchanged on the rest of candidates ${ }^{17}$ in the set $X^{\prime}=\left\{x_{2}, x_{3}, x_{4}\right\}$ under NB and in the set $X^{\prime \prime}=\left\{x_{3}, x_{4}\right\}$ under PB. Then, the PSP will occur if the new elected two-member committee is $C=\left\{x_{2}, x_{4}\right\}$ which means that:

- Under NB over $X^{\prime}=\left\{x_{2}, x_{3}, x_{4}\right\}: x_{2} \mathbf{S} x_{3}$, and $x_{4} \mathbf{S} x_{3}$ under a given rule $\mathbf{S}$.

- Under PB over $X^{\prime \prime}=\left\{x_{3}, x_{4}\right\}: x_{4} \mathbf{S} x_{3}$ under a given $\mathbf{S}$ which is equivalent to $x_{4} \mathbf{M} x_{3}$ in a pairwise comparison between $x_{3}$ and $x_{4}$.

In other words, the PSP will occur under NB (resp. PB) if and only if seven (resp. six) inequalities are satisfied. Using the same general procedure of Section 3, we obtain the volume of each space and the probability of the PSP of each case is provided as a consequence of above remarks. It is obvious that for $k=1$, the corresponding probabilities will be similar for $\mathrm{PB}$ and $\mathrm{NB}$. We denote $P_{P S P}^{N B}(k, m)$ (resp. $P_{P S P}^{P B}(k, m)$ ), the limit probability of the PSP for given values of $k$ and $m$ under NB (resp. PB) in our framework of IAC and large electorates. The summary of our results is displayed in Tables 6-13.

Table 6: $P_{P S P}^{N B}(k, m)$ for $k-$ Plurality and $I A C_{\infty}$

\begin{tabular}{c|cccc}
\hline $\begin{array}{c}m \rightarrow \\
k \downarrow\end{array}$ & 3 & 4 & 5 & 6 \\
\hline 1 & 0.3611 & 0.4135 & 0.4221 & 0.4223 \\
2 & - & 0.2751 & 0.3123 & 0.3443 \\
3 & - & - & 0.2355 & 0.2987 \\
4 & - & - & - & 0.2086
\end{tabular}

Table 7: $P_{P S P}^{P B}(k, m)$ for $k-$ Plurality and $I A C_{\infty}$

\begin{tabular}{c|cccc}
\hline $\begin{array}{c}m \rightarrow \\
k \downarrow\end{array}$ & 3 & 4 & 5 & 6 \\
\hline 1 & 0.3611 & 0.4135 & 0.4221 & 0.4223 \\
2 & - & 0.3773 & 0.4575 & 0.4754 \\
3 & - & - & 0.3820 & 0.4945 \\
4 & - & - & - & 0.3833
\end{tabular}

\footnotetext{
${ }^{16}$ Evidently, for $m=5$ and $m=6$, we are not interested in the ranking neither between candidates in the committee nor between the candidates not in the committee, with the exception of the prior successor.

${ }^{17}$ In other terms, the reduced profile defining the preferences of voters over the rest of candidates will be unchanged in comparison with the original one, with $x_{1}$ removed.
} 
Table 8: $\quad P_{P S P}^{N B}(k, m)$ for $k$-Negative Plurality and $I A C_{\infty}$

\begin{tabular}{c|cccc}
\hline $\begin{array}{c}m \rightarrow \\
k \downarrow\end{array}$ & 3 & 4 & 5 & 6 \\
\hline 1 & 0.1233 & 0.2069 & 0.2750 & 0.3028 \\
2 & - & 0.1582 & 0.2454 & 0.2885 \\
3 & - & - & 0.1836 & 0.2735 \\
4 & - & - & - & 0.1890
\end{tabular}

Table 10: $P_{P S P}^{N B}(k, m)$ for $k$-Borda and $I A C_{\infty}$

\begin{tabular}{c|cccc}
\hline $\begin{array}{c}m \rightarrow \\
k \downarrow\end{array}$ & 3 & 4 & 5 & 6 \\
\hline 1 & 0.1250 & 0.1643 & 0.1731 & 0.1831 \\
2 & - & 0.1218 & 0.1473 & 0.1676 \\
3 & - & - & 0.1063 & 0.1507 \\
4 & - & - & - & 0.0979
\end{tabular}

Table 12: $P_{P S P}^{N B}(k, m)$ for Bloc and $I A C_{\infty}$

\begin{tabular}{c|cccc}
\hline $\begin{array}{c}m \rightarrow \\
k \downarrow\end{array}$ & 3 & 4 & 5 & 6 \\
\hline 1 & 0.3611 & 0.4135 & 0.4221 & 0.4223 \\
2 & - & 0.3130 & 0.3513 & 0.3923 \\
3 & - & - & 0.2741 & 0.3321 \\
4 & - & - & - & 0.2380
\end{tabular}

Table 9: $\quad P_{P S P}^{P B}(k, m)$ for $k$-Negative Plurality and $I A C_{\infty}$

\begin{tabular}{c|cccc}
\hline $\begin{array}{c}m \rightarrow \\
k \downarrow\end{array}$ & 3 & 4 & 5 & 6 \\
\hline 1 & 0.1233 & 0.2069 & 0.2750 & 0.3028 \\
2 & - & 0.2455 & 0.3722 & 0.3958 \\
3 & - & - & 0.3360 & 0.4774 \\
4 & - & - & - & 0.3658
\end{tabular}

Table 11: $P_{P S P}^{P B}(k, m)$ for $k$-Borda and $I A C_{\infty}$

\begin{tabular}{c|cccc}
\hline $\begin{array}{c}m \rightarrow \\
k \downarrow\end{array}$ & 3 & 4 & 5 & 6 \\
\hline 1 & 0.1250 & 0.1643 & 0.1731 & 0.1831 \\
2 & - & 0.1947 & 0.2498 & 0.2775 \\
3 & - & - & 0.2220 & 0.3037 \\
4 & - & - & - & 0.2422
\end{tabular}

Table 13: $P_{P S P}^{P B}(k, m)$ for Bloc and $I A C_{\infty}$

\begin{tabular}{c|cccc}
\hline $\begin{array}{c}m \rightarrow \\
k \downarrow\end{array}$ & 3 & 4 & 5 & 6 \\
\hline 1 & 0.3611 & 0.4135 & 0.4221 & 0.4223 \\
2 & - & 0.2990 & 0.3894 & 0.4434 \\
3 & - & - & 0.2913 & 0.4795 \\
4 & - & - & - & 0.3258
\end{tabular}

Several lessons may be drawn from these probabilities.

- Our results support the superiority of $k$-Borda when the goal is to minimize the probability of the PSP, with the exception of $m=3$ and $k=1$ where $k$-Negative Plurality performs better.

- Bloc appears to be more vulnerable to the PSP than $k$-Plurality and $k$-Negative Plurality.

- The likelihood of the PSP is lower when considering NB than PB with independence of the analysed multi-winner voting rule.

- With NB, the probability of the PSP decreases when $k$ increases. This fact hold with independence of the assumed voting rule. However, we can not draw a clear conclusion about the relationship between our probabilities and the parameter $k$ with PB. 


\subsection{The leaving member paradox}

Recall the conditions for the LMP to occur. Voters use a multi-winner voting method in order to elect a committee of size $k$ out of $m$ candidates in the set $X$. A candidate $x_{1}$ drops out of the competition for some reasons. If a new election holds, a new committee can be elected while neither of the original committee members are considered. In other words, the new committee and the one corresponding to the original election are disjoint. The previous observations make it clear that the LMP only occurs under NB since, under $\mathrm{PB}$, we can not end up with a committee totally different from what we have in the original election. In addition, the LMP is clearly not defined with less than four candidates and $k$ greater than $\left\lfloor\frac{m}{2}\right\rfloor$, where \lfloor\rfloor stands for the integer part of the number.

Following the logic of the previous analysis, the limiting probabilities of the LMP are found no matter the ranking of the leaving member. Again, we suppose that the reduced preferences over the remaining candidates will be unchanged, when a candidate leaves. We denote by $P_{L M P}^{N B}(k, m)$ the probability of the LMP in our framework of IAC when $n$ tends to infinity. Table 14 lists these probabilities for $2 \leq k \leq\left\lfloor\frac{m}{2}\right\rfloor$ with $4 \leq m \leq 6$, and the results suggest that:

- $k$-Borda has a performance that is superior to the other multi-winner rules over the range of all possible values of $k$ and $m$.

- Bloc appears to exhibit extremely poor performance than $k$-Plurality and $k$-Negative Plurality.

- For a fixed value of $m$, the probability of the LMP decreases significantly and eventually tends to zero as $k$ increases.

Table 14: $P_{L M P}^{N B}(k, m)$ under $I A C_{\infty}$

\begin{tabular}{r|c|ccc}
\hline & $m \rightarrow$ & 4 & 5 & 6 \\
$k \downarrow$ & & & \\
\hline \multirow{2}{*}{$k$-Plurality } & 2 & 0.0485 & 0.0714 & 0.0751 \\
& 3 & - & - & 0.0012 \\
\hline \multirow{2}{*}{$k$-Negative Plurality } & 2 & 0.0286 & 0.0465 & 0.0667 \\
& 3 & - & - & 0.0005 \\
\hline \multirow{2}{*}{$k$-Borda } & 2 & 0.0094 & 0.0144 & 0.0172 \\
& 3 & - & - & 0.0000 \\
\hline \multirow{2}{*}{ Bloc } & 2 & 0.0757 & 0.0891 & 0.1024 \\
& 3 & - & - & 0.0020
\end{tabular}

\section{Concluding remarks}

In this paper, we have conducted a comparison of four multi-winner scoring rules on the basis of two criteria. First, the ability to select the Condorcet committee à la Gehrlein when it exists, and second the likelihood of both the prior successor paradox and the leaving member paradox. The choice of the voting rules was guided by the fact that each of them is based on some single-winner scoring rule. It turns out that, among all the rules 
that we consider, $k$-Borda performs better. It also comes from our results that, in many cases, Bloc performs quite badly comparatively to $k$-Plurality and $k$-Negative Plurality. Moreover, we believe that our results give a better understanding of how the likelihood of the paradoxes change as the target size of the committee and the number of candidates vary.

As noticed in the Introduction, another generalization of a Condorcet winner to committees is the one proposed by Fishburn (1981). In contrast to Gehrlein (1985b), there, it is assumed that the voters have preferences over committees that satisfy certain conditions. In particular, this approach defines a Condorcet committee as a committee that is preferred to every other committee by a majority of voters. Notice that, Hill (1988), Kaymak and Sanver (2003), and Kamwa and Merlin (2013) examined under which condition a Condorcet committee in the sense of Fishburn coincide with the one in the sense of Gehrlein. Obviously, the answer depends on the extension function used for lifting individual preferences from alternatives to subsets. Other approaches (e.g. Brams et al. (2006), Ratliff (2003, 2006), Elkind et al. (2011), among others) are taken in the literature and can be considered as closely related to the problems we consider. We believe that studying other variants of the Condorcet committee is an important research direction.

After comparing the four scoring-based rules that we consider in our paper, it is tempting to simply study other multi-winner rules. Perhaps, a better idea would be to consider procedures based on repeated ballots and elimination of one (or more) candidate in each round, called multi-stage or sequential elimination voting rules. Here, we can imagine that the process will continue to reduce the number of candidates to $k$ candidates, the target size of the committee. Then, the challenge would be to study the impact of multi-stage voting on the probability of the paradoxes.

\section{References}

S. Berg and D. Lepelley. On probability models in voting theory. Statistica Neerlandica, 48 (2):133-146, 1994.

S. Brams, D. Kilgour, and M. Sanver. How to elect a representative committee using Approval balloting, pages 83-95. In: Simeone, B. and Pukelsheim, F. (eds.), Mathematics and Democracy. Studies in Choice and Welfare, Springer, Berlin, 2006.

S.J. Brams. Approval Voting in multicandidate elections. Policy Studies Journal, 9:102108, 1980.

S.J. Brams and P.C. Fishburn. Approval Voting. American Political Science Review, 72: 831-847, 1978.

S.J. Brams and P.C. Fishburn. Going from theory to practice: The mixed success of Approval Voting. Social Choice and Welfare, 25:457-474, 2005.

B. Bueler, A. Enge, and K. Fukuda. Exact volume computation for polytopes: A practical study. Polytopes - Combinatorics and Computation, DMV Seminar, 29:131-154, 2000.

D. Cervone, W.V. Gehrlein, and W. Zwicker. Which scoring rule maximizes Condorcet efficiency under IAC? Theory and Decision, 58:145-185, 2005.

J. Cohen and T. Hickey. Two algorithms for determining volumes of convex polyhedra. Journal of the ACM, 26:401-414, 1979. 
Marquis de Condorcet. Essai sur l'application de l'analyse à la probabilité des décisions rendues à la pluralité des voix. Paris, 1785.

B. Debord. Prudent $k$-choice functions: Properties and algorithms. Mathematical Social Sciences, 26:63-77, 1993.

M. Diss and W.V. Gehrlein. Borda's paradox with weighted scoring rules. Social Choice and Welfare, 38:121-136, 2012.

M. Diss and W.V. Gehrlein. The true impact of voting rule selection on Condorcet efficiency. Economics Bulletin, 35:2418-2426, 2015.

M. Diss, V. Merlin, and F. Valognes. On the Condorcet efficiency of Approval Voting and extended scoring rules for three alternatives, pages 255-283. In: Handbook of Approval Voting, Sanver and Laslier (eds). Heidelberg; Springer-Verlag, 2010.

E. Elkind, J. Lang, and A. Saffidine. Choosing collectively optimal sets of alternatives based on the Condorcet criterion. In IJCAI'11, pages 186-191, 2011.

E. Elkind, P. Faliszewski, P. Skowron, and A. Slinko. Properties of multiwinner voting rules. Mimeo, 2015.

D.S. Felsenthal and Z. Maoz. Normative properties of four single-stage multi-winner electoral procedures. Behavioral Science, 37:109-127, 1992.

P.C. Fishburn. Simple voting systems and majority rule. Behavioral Science, 19:166-179, 1974a.

P.C. Fishburn. Aspects of one-stage voting rules. Management Science, 21:422-427, 1974b.

P.C. Fishburn. An analysis of simple voting systems for electing committees. SIAM Journal on Applied Mathematics, 41:499-502, 1981.

P.C. Fishburn and S.J. Brams. Approval Voting, Condorcet's principle, and runoff elections. Public Choice, 36:89-114, 1981.

P.C. Fishburn and W.V. Gehrlein. An analysis of simple two stage voting systems. Behavioral Science, 21:1-12, 1976a.

P.C. Fishburn and W.V. Gehrlein. Borda's rule, positional voting, and Condorcet's simple majority principle. Public Choice, 28:79-88, 1976b.

P.C. Fishburn and W.V. Gehrlein. An analysis of voting procedures with non-ranked voting. Public Choice, 22:178-185, 1977.

E. Gawrilow and M. Joswig. Polymake: A framework for analyzing convex polytopes. In Gil Kalai and Günter M. Ziegler, editors, Polytopes - Combinatorics and Computation, pages 43-74. Birkhäuser, 2000.

W.V. Gehrlein. Condorcet efficiency of constant scoring rules for large electorates. Economics Letters, 19:13-15, 1985a.

W.V. Gehrlein. The Condorcet criterion and committee selection. Mathematical Social Sciences, 10:199-209, 1985b. 
W.V. Gehrlein. Condorcet efficiency and social homogeneity, pages 127-143. In: Barnett, Moulin, Salles, Schofield (eds). Social choice, welfare and ethics. Cambridge University Press, Cambridges, 1995.

W.V. Gehrlein. The Condorcet efficiency of Borda rule under the Dual Culture condition. Social Science Research, 28:36-44, 1999.

W.V. Gehrlein. Condorcet winners on four candidates with anonymous voters. Economics Letters, 71:335-340, 2001.

W.V. Gehrlein and P.C. Fishburn. Condorcet's paradox and anonymous preference profiles. Public Choice, 26:1-18, 1976.

W.V. Gehrlein and D. Lepelley. Condorcet efficiencies under the Maximal Culture condition. Social Choice and Welfare, 16:471-490, 1999.

W.V. Gehrlein and D. Lepelley. The Condorcet efficiency of Borda rule with anonymous voters. Mathematical Social Sciences, 41:39-50, 2001.

W.V. Gehrlein and D. Lepelley. Voting paradoxes and group coherence: The Condorcet efficiency of voting rules. Springer, 2010.

W.V. Gehrlein and D. Lepelley. The value of research based on simple assumptions about voters' preferences, pages 173-200. In: DS Felsenthal, M Machover (eds). Electoral systems: Paradoxes, assumptions and procedures. Publisher: Springer-Verlag, 2012.

W.V. Gehrlein and D. Lepelley. The Condorcet efficiency advantage that voter indifference gives to Approval Voting over some other voting rules. Group Decision and Negotiation, 24:243-269, 2015.

G.T. Guilbaud. Les théories de l'intérêt général et le problème logique de l'agrégation. Economie Appliquée, 5:501-584, 1952.

I.D. Hill. Some aspects of elections: To fill one seat or many. Journal of the Royal Statistical Society, 151:243-275, 1988.

E. Kamwa. The Increasing Committee Size Paradox with a small number of candidates. Economics Bulletin, 33:967-972, 2013.

E. Kamwa and V. Merlin. Coincidence of Condorcet committees. Normandie Univ, Mimeo, 2013.

E. Kamwa and V. Merlin. Scoring rules over subsets of alternatives: Consistency and paradoxes. Journal of Mathematical Economics, 61:130-138, 2015.

B. Kaymak and R. Sanver. Sets of alternatives as Condorcet winners. Social Choice and Welfare, 20:477-494, 2003.

J. Lawrence. Polytope volume computation. Mathematics of Computation, 57:259-271, 1991.

D. Lepelley, A. Louichi, and F. Valognes. Computer simulations of voting systems. Advances in Complex Systems, 03:181-194, 2000a.

D. Lepelley, P. Pierron, and F. Valognes. Scoring rules, Condorcet efficiency and social homogeneity. Theory and Decision, 49:175-196, 2000b. 
D. Lepelley, A. Louichi, and H. Smaoui. On Ehrhart polynomials and probability calculations in voting theory. Social Choice and Welfare, 30:363-383, 2008.

S. Merrill. A comparison of efficiencies of multicandidate electoral systems. American Journal of Political Science, 28:23-84, 1984.

S. Merrill. A statistical model for Condorcet efficiency based on simulation under spatial model assumptions. Public Choice, 47:389-403, 1985.

D.W. Mitchell and W.N. Trumbull. Frequency of paradox in a common $n$-winner voting scheme. Public Choice, 73:55-69, 1992.

J. Pérez, J.-L. Jimeno, and E. García. No Show paradox in Condorcet $k$-voting procedures. Group Decision and Negotiation, 21:291-303, 2012.

T. Ratliff. Some startling inconsistencies when electing committees. Social Choice and Welfare, 21:433-454, 2003.

T. Ratliff. Selecting committees. Public Choice, 126:343-355, 2006.

M. Staring. Two paradoxes of committee elections. Mathematics Magazine, 59:158-159, 1986.

H. Young. Social choice scoring functions. SIAM Journal on Applied Mathematics, 28: 824-838, 1975. 